\title{
Tomography of a Polymer/Carbon-Nanotube Matrix Composite
}

\author{
G.B. Thompson*, M. Abdalla** and D. Dean**
}

* University of Alabama, Department of Metallurgical \& Materials Engineering, $2017^{\text {th }}$ Ave, A129 Bevill Building, Tuscaloosa, AL 35487-0202

** University of Alabama at Birmingham, Department of Materials Science \& Engineering, BEC 254, $15303^{\text {rd }}$ Ave. South, Birmingham, AL 35294-4461

Nanocomposites based on carbon nanotubes (CNTs) have received a tremendous amount of attention during the past five years. Individually, CNTs can achieve very high aspect ratios because their diameters are in the range of a few nanometers with lengths of several hundred nanometers. Additionally, CNTs are reported to have extremely high elastic modulus, $\sim 1 \mathrm{TPa}$ which is comparable to that of diamond (1.2 TPa). The strength of CNTs is 10-100 times that of the strongest steel but at a fraction of the weight [1-4]. CNTs exhibit a range of electrical conductivity, from metallic to moderate band gap semiconductors depending on their structure [5]. Potential applications of polymer/CNT composites are in aerospace (high temperature, light weight), automobile (bumpers, interior and exterior panels, gasoline tanks), construction (panels), electronic and electrical (printed circuits, electric component), packaging (films, containers), adhesives and coatings. In spite of a CNT's outstanding individual properties, the elastic modulus of nanotube based composites is low; e.g., Shaffer and Windle [3] reported that the Young's modulus value of a CNT-PVA composite film was $\sim 150 \mathrm{MPa}$. A main obstacle in improving the stiffness and strength of a CNT composite is the control of the nanotube's dispersion, alignment and load transfer at the interfaces within the matrix.

We have addressed these issues in the present study using a combination of surface modification and processing techniques to prepare epoxy/CNT nanocomposites which have a controlled spatial distribution of the CNTs. The surfaces of the multiwall carbon nanotubes(MWCNTs) were chemically modified to enhance interaction with the polymer, which was an epoxy resin. We used mechanical shearing to align the carbon nanotubes as well. Furthermore, we believe that the high shear coupled with the modified surface helps to prevent aggregation of the nanotubes.

To determine how the layered morphology of our nanotubes in the polymer contributed to the mechanical properties, 3-dimensional characterization of the CNT filler in the polymer matrix is critical. Recent advances in High Angle Annular Dark Field-Scanning Transmission Electron Microscopy (HAADF-STEM) allow images to be taken at various angles with minimal BraggDiffraction contrast contributions to the image [6].

We have performed a single tilt series for a series of polymer/nanotube composites using a FEI Tecnai F20 Supertwin Transmission Electron Microscope (TEM). The images were taken for tilt angles between $+/-50^{\circ}$ to $+/-70^{\circ}$ with $1^{\circ}$ step intervals. The images were then compiled and reconstructed using FEI's Xplore $3 \mathrm{D}{ }^{\circledR}$ and Inspect3D ${ }^{\circledR}$ imaging and Amira ${ }^{\circledR}$ software. The TEM foils were prepared by cutting thin slices from the composite using standard microtone techniques and laying each slice onto a TEM carbon supported film. As evident in Fig. 1, the tomography images clearly provide new viewing prospective to determine the extent of layering and directionality of the filler tubes in the polymer. From this figure, we can see groups of tubes aligned 
in the same direction. From the bright field TEM images, some of these tubes appear to be significantly bent. By using tomography, we will address how different processing and mechanical testing results in variations of CNTs distribution and directionality within the polymer matrix.

\section{References:}

[1] L. Forri, J-P Salvetat, J-M Bonard, R Basca, N. H. Thomson, S. Garaj, L. Thien-Nag, R. Gaál, A. Kulik, B. Ruzicka, L. Degiorgi, A. Bachtold, C. Schönenberger, S. Pekker, K. Hernadi "Electronic and Mechanical Properties of Carbon Nanotubes", Science and Application of Nanotubes, pp. 297, Edited by

[2] D. Tomànek and R.J. Enbody, Kluwer Academic / Plenum Publishers, New York, 2000

[3] M.S.P. Shaffer and A.H. Windle, Fabrication and Characterization of Carbon

Nanotube/Poly(vinyl alcohol) Composites, Advanced Materials 1999, 11, pp. 937-941

[4] S. J. V. Frankland, A. Caglar, D. W. Brenner, and M. Griebel, Reinforcement mechanisms in polymer nanotube composites: Simulated non-bonded and cross-linked systems, Mat. Res. Soc. Symp. Proc., 633, 2000, pp. A. 14.17.1-5.

[5] X. Gong, J.Liu, S.Baskaran, R.D.Voise, J.S.Young, "Surfactant-Assisted Processing of Carbon Nanotube / Polymer Composites," Chem. Mater. 2000, 12, pp. 1049-1052.

[6] P. A. Midgley, M. Weyland, J. M. Thomas and B. F. G. Johnson Chem. Commun. 10 (2001) 907.

[7] The authors gratefully acknowledge NSF-MRI-\#0421376 for the acquisition of the TEM used in this research.

(a)

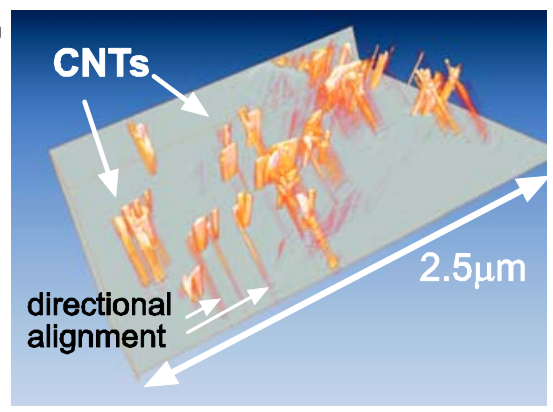

(b)
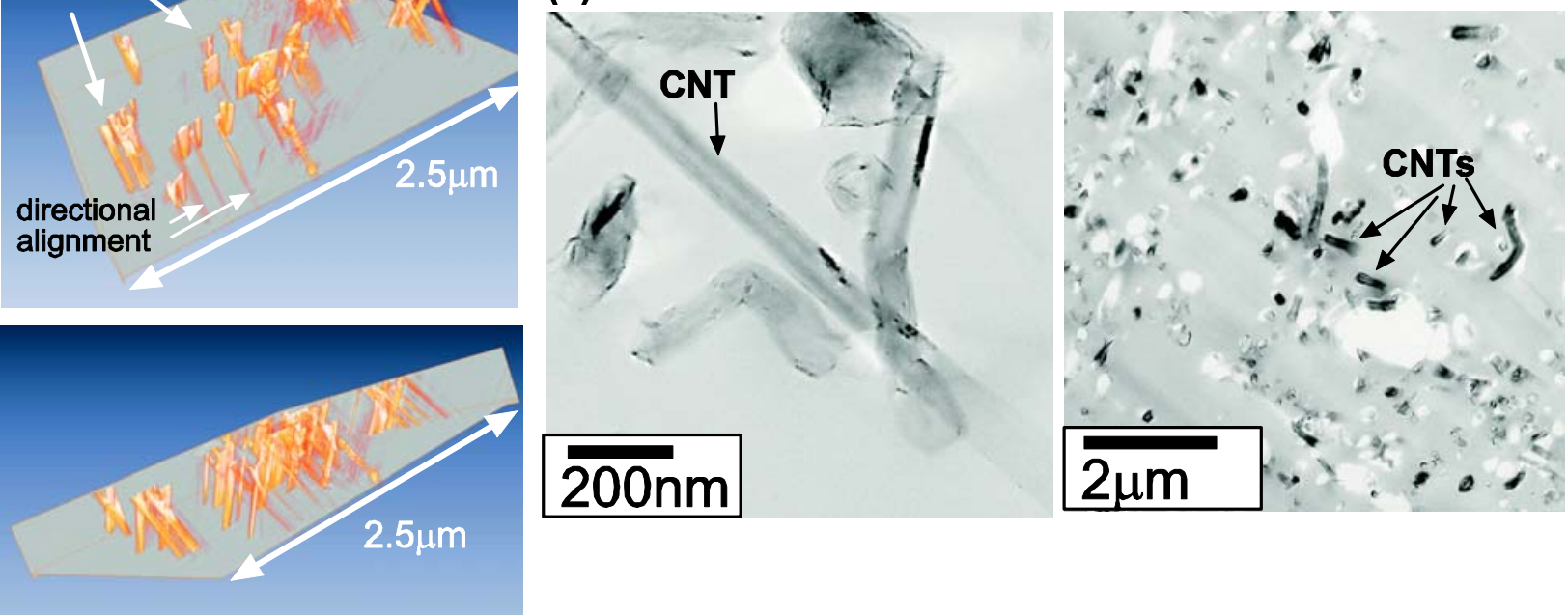

Fig 1: (a) Different viewing angles collected from a HAADF-STEM tomography reconstruction (b) Bright Field TEM images of the specimen. 